\title{
Wiener-Hopf Operators in Higher Dimensions: The Widom Conjecture for Piece-Wise Smooth Domains
}

\author{
A. V. Sobolev
}

\begin{abstract}
We prove a two-term quasi-classical trace asymptotic formula for the functions of multi-dimensional Wiener-Hopf operators with discontinuous symbols. The discontinuities occur on surfaces which are assumed to be piece-wise smooth. Such a two-term formula was conjectured by $\mathrm{H}$. Widom (On a Class of Integral Operators with Discontinuous Symbol, Toeplitz centennial (Tel Aviv, 1981), pp. 477-500. Operator Theory: Advances and Applications, vol. 4. Birkhäuser, Basel, 1982), and proved by A. V. Sobolev for smooth surfaces in 2009 (Mem. AMS 222(1043), 2013).
\end{abstract}

Mathematics Subject Classification. Primary 47G30, 35S05; Secondary 45M05, 47B10, 47B35.

Keywords. Wiener-Hopf operators, Pseudo-differential operators with discontinuous symbols, Quasi-classical asymptotics.

\section{Introduction}

The quasi-classical functional calculus for smooth pseudo-differential operators was developed more than three decades ago (see e.g. [5]) and now it is considered a standard tool of microlocal analysis and spectral theory. On the contrary, for pseudo-differential operators with discontinuous symbols results are sparse and less well known. Various quasi-classical trace type formulas for Wiener-Hopf operators were obtained by H. Widom in the 80's. In this article we shall be concerned with a multi-dimensional generalisation of one such result which has become known as The Widom Conjecture. Let $a=a(\mathbf{x}, \boldsymbol{\xi}), \mathbf{x}, \boldsymbol{\xi} \in \mathbb{R}^{d}, d \geq 1$ be a smooth symbol. Introduce the standard notation for the left and right pseudo-differential operators with symbol $a$ and a quasi-classical parameter $\alpha>0$ :

$$
\left(\mathrm{Op}_{\alpha}^{1}(a) u\right)(\mathbf{x})=\left(\frac{\alpha}{2 \pi}\right)^{d} \iint e^{i \alpha(\mathbf{x}-\mathbf{y}) \boldsymbol{\xi}} a(\mathbf{x}, \boldsymbol{\xi}) u(\mathbf{y}) d \boldsymbol{\xi} d \mathbf{y}
$$




$$
\left(\mathrm{Op}_{\alpha}^{\mathrm{r}}(a) u\right)(\mathbf{x})=\left(\frac{\alpha}{2 \pi}\right)^{d} \iint e^{i \alpha(\mathbf{x}-\mathbf{y}) \boldsymbol{\xi}} a(\mathbf{y}, \boldsymbol{\xi}) u(\mathbf{y}) d \boldsymbol{\xi} d \mathbf{y}
$$

for any function $u$ from the Schwartz class on $\mathbb{R}^{d}$. If the function $a$ depends only on $\boldsymbol{\xi}$ then the operators $\mathrm{Op}_{\alpha}^{\mathrm{l}}(a), \mathrm{Op}_{\alpha}^{\mathrm{r}}(a)$ coincide with each other, and we simply write $\mathrm{Op}_{\alpha}(a)$. Here and below integrals without indication of the domain are assumed to be taken over the entire Euclidean space $\mathbb{R}^{d}$.

Let $\Lambda, \Omega$ be bounded domains in $\mathbb{R}^{d}$, and let $\chi_{\Lambda}, \chi_{\Omega}$ be their characteristic functions, $P_{\Omega, \alpha}=\mathrm{Op}_{\alpha}\left(\chi_{\Omega}\right)$. We are interested in spectral properties of the operators

$$
T_{\alpha}(a)=T_{\alpha}(a ; \Lambda, \Omega)=\chi_{\Lambda} P_{\Omega, \alpha} \operatorname{Op}_{\alpha}^{1}(a) P_{\Omega, \alpha} \chi_{\Lambda},
$$

and

$$
S_{\alpha}(a)=S_{\alpha}(a ; \Lambda, \Omega)=\chi_{\Lambda} P_{\Omega, \alpha} \operatorname{ReOp}_{\alpha}^{1}(a) P_{\Omega, \alpha} \chi_{\Lambda}
$$

These operators are naturally interpreted as multi-dimensional Wiener-Hopf operators with discontinuous symbols. Our aim is to find asymptotic formulas for the traces of the form $\operatorname{tr} g\left(T_{\alpha}\right), \operatorname{tr} g\left(S_{\alpha}\right)$ as $\alpha \rightarrow \infty$, with suitable functions $g, g(0)=0$. If one of the domains, e.g. $\Omega$, coincides with $\mathbb{R}^{d}$ then assuming that $a$ is infinitely differentiable and decays sufficiently fast, one can write out complete asymptotic expansions of the above traces in powers of $\alpha^{-1}$, see [9]. Our main focus will be on the case when both domains $\Lambda$ and $\Omega$ are distinct from $\mathbb{R}^{d}$. The Widom Conjecture states (see [8]) that in this case

$$
\operatorname{tr} g\left(T_{\alpha}\right)=\alpha^{d} \mathfrak{W}_{0}+\alpha^{d-1} \log \alpha \mathfrak{W}_{1}+o\left(\alpha^{d-1} \log \alpha\right),
$$

as $\alpha \rightarrow \infty$. The precise formulas for the coefficients $\mathfrak{W}_{0}, \mathfrak{W}_{1}$ are given in Sect. 2. The first term in (1.3) is the standard Weyl asymptotics, whereas the second term is non-standard, and it describes the contribution of the boundaries $\partial \Lambda, \partial \Omega$. Emphasise that the second term contains a log-factor which makes it different from the familiar asymptotic expansion in powers of $\alpha^{-1}$. The formula (1.3) was proved by H. Widom in [8] for $d=1$. For $d \geq 2$, in the case when one of the domains is a half-space, (1.3) was justified in [10]. For arbitrary bounded smooth domains in $\mathbb{R}^{d}, d \geq 2$, the conjecture was proved in $[6]$.

The main aim of this paper is to extend (1.3) to piece-wise smooth domains. Apart from the purely mathematical motivation, the interest in such domains is dictated by applications in Mathematical Physics, and in particular in Quantum Information Theory, see [2-4]. The proof is based on the papers $[6,7]$. Using a convenient partition of unity one separates contributions from the smooth and non-smooth parts of the boundaries $\partial \Lambda$ and $\partial \Omega$. For the smooth part one applies directly the local version of the asymptotic formula of the form (1.3) from [6], whereas for the non-smooth part it suffices to establish appropriate trace bounds. Here a key role is played by inequalities obtained for arbitrary Lipschitz domains in [7]. As a result one checks that the non-smooth portion of the boundaries contributes a term of size $o\left(\alpha^{d-1} \log \alpha\right)$, which leads to the global asymptotics (1.3). 


\section{Main Results}

We begin with describing the classes of domains with which we work. In what follows we always assume that $d \geq 2$.

Definition 2.1. 1. We say that $\Lambda \subset \mathbb{R}^{d}$ is a basic Lipschitz domain (resp. basic $\mathrm{C}^{m}$-domain, $\left.m=1,2, \ldots\right)$ if there exists a Lipschitz (resp. $\mathrm{C}^{m}$ ) function $\Phi=\Phi(\hat{\mathbf{x}}), \hat{\mathbf{x}} \in \mathbb{R}^{d-1}$, such that with a suitable choice of the Cartesian coordinates $\mathbf{x}=\left(\hat{\mathbf{x}}, x_{d}\right), \hat{\mathbf{x}}=\left(x_{1}, x_{2}, \ldots, x_{d-1}\right)$ the domain $\Lambda$ is represented as

$$
\Lambda=\left\{\mathbf{x} \in \mathbb{R}^{d}: x_{d}>\Phi(\hat{\mathbf{x}})\right\} .
$$

For a basic Lipschitz domain the function $\Phi$ is assumed to be uniformly Lipschitz, i.e. the constant

$$
M=M_{\Phi}=\sup _{\substack{\hat{\mathbf{x}}, \hat{\mathbf{y}}, \hat{\mathbf{x}} \neq \hat{\mathbf{y}}}} \frac{|\Phi(\hat{\mathbf{x}})-\Phi(\hat{\mathbf{y}})|}{|\hat{\mathbf{x}}-\hat{\mathbf{y}}|}
$$

is finite. For a basic $C^{m}$-domain all the derivatives $\nabla^{n} \Phi, n=1,2, \ldots, m$, are assumed to be uniformly bounded on $\mathbb{R}^{d-1}$. For a basic domain we use the notation $\Lambda=\Gamma(\Phi)$.

2. A domain $\Lambda \subset \mathbb{R}^{d}$ is said to be Lipschitz (resp. $\left.\mathrm{C}^{m}, m=1,2, \ldots\right)$ if $\Lambda \neq \mathbb{R}^{d}$ and locally it can be represented by basic Lipschitz (resp. $\mathrm{C}^{m}$-) domains, i.e. for any $\mathbf{z} \in \Lambda$ there is a radius $r>0$ such that $B(\mathbf{z}, r) \cap \Lambda=B(\mathbf{z}, r) \cap \Lambda_{0}$ with some basic Lipschitz (resp. $\mathrm{C}^{m}$-) domain $\Lambda_{0}=\Lambda_{0}(\mathbf{z})$ or with $\Lambda_{0}=\mathbb{R}^{d}$. In this case the boundary $\partial \Lambda$ is said to be a $(d-1)$-dimensional Lipschitz (resp. $C^{m}$-) surface.

3. A basic Lipschitz domain $\Lambda=\Gamma(\Phi)$ is said to be piece-wise $\mathrm{C}^{m}$ with some $m=1,2, \ldots$, if the function $\Phi$ is $C^{m}$-smooth away from a collection of finitely many $(d-2)$-dimensional Lipschitz surfaces $L_{1}, L_{2}, \ldots, L_{n} \subset$ $\mathbb{R}^{d-1}$. More precisely, if $\Lambda$ is given by (2.1) then for any open ball $B \subset$ $\mathbb{R}^{d-1}$ such that $\bar{B}$ is disjoint with all surfaces $L_{j}, j=1,2, \ldots, n$, we have $\Phi \in \mathrm{C}^{m}(\bar{B})$. Note that the derivatives of $\Phi$ are not required to be bounded uniformly in the choice of the ball $B$. We denote

$$
(\partial \Lambda)_{\mathrm{s}}=\left\{\mathbf{x}=(\hat{\mathbf{x}}, \Phi(\hat{\mathbf{x}})), \hat{\mathbf{x}} \in \bigcup_{j=1}^{n} L_{j}\right\}
$$

i.e. $(\partial \Lambda)_{\mathrm{s}} \subset \partial \Lambda$ is the set of points where the $C^{m}$-smoothness of the surface $\partial \Lambda$ may break down.

4. A Lipschitz domain $\Lambda$ is said to be piece-wise $\mathrm{C}^{m}, m=1,2, \ldots$, if locally it can be represented by piece-wise $C^{m}$ basic domains. As for the basic domains, by $(\partial \Lambda)_{\mathrm{s}} \subset \partial \Lambda$ we denote the set of points where the $\mathrm{C}^{m}$-smoothness of $\partial \Lambda$ may break down.

Let us define the asymptotic coefficients entering the main asymptotic formulas. For a symbol $b=b(\mathbf{x}, \boldsymbol{\xi})$ let

$$
\mathfrak{W}_{0}(b)=\mathfrak{W}_{0}(b ; \Lambda, \Omega)=\frac{1}{(2 \pi)^{d}} \int_{\Lambda} \int_{\Omega} b(\mathbf{x}, \boldsymbol{\xi}) d \boldsymbol{\xi} d \mathbf{x} .
$$


For any $(d-1)$-dimensional Lipschitz surfaces $L, P$ denote

$$
\mathfrak{W}_{1}(b)=\mathfrak{W}_{1}(b ; L, P)=\frac{1}{(2 \pi)^{d-1}} \int_{L} \int_{P} b(\mathbf{x}, \boldsymbol{\xi})\left|\mathbf{n}_{L}(\mathbf{x}) \cdot \mathbf{n}_{P}(\boldsymbol{\xi})\right| d S_{\boldsymbol{\xi}} d S_{\mathbf{x}},
$$

where $\mathbf{n}_{L}(\mathbf{x})$ and $\mathbf{n}_{P}(\boldsymbol{\xi})$ denote the exterior unit normals to $L$ and $P$ defined for a.a. $\mathbf{x}$ and $\boldsymbol{\xi}$ respectively. For any continuous function $g$ on $\mathbb{C}$ such that $g(0)=0$, and any number $s \in \mathbb{C}$, we also define

$$
\mathfrak{A}(g ; s)=\frac{1}{(2 \pi)^{2}} \int_{0}^{1} \frac{g(s t)-t g(s)}{t(1-t)} d t .
$$

The next theorem contains the main result of the paper.

Theorem 2.2. Let $\Lambda, \Omega \subset \mathbb{R}^{d}, d \geq 2$ be bounded Lipschitz domains in $\mathbb{R}^{d}$ such that $\Lambda$ is piece-wise $\mathrm{C}^{1}$ and $\Omega$ is piece-wise $\mathrm{C}^{3}$. Let $a=a(\mathbf{x}, \boldsymbol{\xi})$ be a symbol whose distributional derivatives satisfy the bounds

$$
\max _{\substack{0 \leq n \leq d+2 \\ 0 \leq m \leq d+2}} \operatorname{ess-sup}\left|\nabla_{\mathbf{x}, \boldsymbol{\xi}}^{n} \nabla_{\boldsymbol{\xi}}^{m} a(\mathbf{x}, \boldsymbol{\xi})\right|<\infty .
$$

Let $g$ be a function on $\mathbb{C}$ such that $g(0)=0$, analytic in a disk of sufficiently large radius. Then

$$
\begin{aligned}
\operatorname{tr} g\left(T_{\alpha}(a)\right)= & \alpha^{d} \mathfrak{W}_{0}(g(a) ; \Lambda, \Omega)+\alpha^{d-1} \log \alpha \mathfrak{W}_{1}(\mathfrak{A}(g ; a) ; \partial \Lambda, \partial \Omega) \\
& +o\left(\alpha^{d-1} \log \alpha\right)
\end{aligned}
$$

as $\alpha \rightarrow \infty$.

For the self-adjoint operator $S_{\alpha}(a)$ we have a wider choice of functions $g$ :

Theorem 2.3. Let the domains $\Lambda, \Omega \subset \mathbb{R}^{d}, d \geq 2$, and the symbol a be as in Theorem 2.2. Then for any function $g \in C^{\infty}(\mathbb{R})$, such that $g(0)=0$, one has

$$
\begin{aligned}
\operatorname{tr} g\left(S_{\alpha}(a)\right)= & \alpha^{d} \mathfrak{W}_{0}(g(\operatorname{Re} a) ; \Lambda, \Omega)+\alpha^{d-1} \log \alpha \mathfrak{W}_{1}(\mathfrak{A}(g ; \operatorname{Re} a) ; \partial \Lambda, \partial \Omega) \\
& +o\left(\alpha^{d-1} \log \alpha\right),
\end{aligned}
$$

as $\alpha \rightarrow \infty$.

As in [6] the crucial step of the proof is to prove the formula (2.7) for polynomial functions.

Theorem 2.4. Let the domains $\Lambda, \Omega \subset \mathbb{R}^{d}, d \geq 2$, and the symbol a be as in Theorem 2.2. Then for $g_{p}(t)=t^{p}, p=1,2, \ldots$, we have

$$
\begin{aligned}
\operatorname{tr} g_{p}\left(T_{\alpha}(a)\right)= & \alpha^{d} \mathfrak{W}_{0}\left(g_{p}(a) ; \Lambda, \Omega\right)+\alpha^{d-1} \log \alpha \mathfrak{W}_{1}\left(\mathfrak{A}\left(g_{p} ; a\right) ; \partial \Lambda, \partial \Omega\right) \\
& +o\left(\alpha^{d-1} \log \alpha\right),
\end{aligned}
$$

as $\alpha \rightarrow \infty$. If $T_{\alpha}(a)$ is replaced with $S_{\alpha}(a)$, then the same formula holds with the symbol a replaced by $\operatorname{Re} a$ on the right-hand side.

In the next theorem the domain $\Lambda$ is allowed to be unbounded, in which case we replace formula (2.9) with its regularized variant. 
Theorem 2.5. Let $\Lambda, \Omega \subset \mathbb{R}^{d}, d \geq 2$ be Lipschitz domains in $\mathbb{R}^{d}$ such that

1. $\Omega$ is bounded and piece-wise $\mathrm{C}^{3}$,

2. $\Lambda$ or $\mathbb{R}^{d} \backslash \Lambda$ is bounded, and $\Lambda$ is piece-wise $\mathrm{C}^{1}$.

Let the symbol $a$ be as in Theorem 2.2. Then

$$
\begin{aligned}
\lim _{\alpha \rightarrow \infty} \frac{1}{\alpha^{d-1} \log \alpha} \operatorname{tr}\left[g_{p}\left(T_{\alpha}(a ; \Lambda, \Omega)\right)\right. & \left.-\chi_{\Lambda} g_{p}\left(T_{\alpha}\left(a ; \mathbb{R}^{d}, \Omega\right)\right) \chi_{\Lambda}\right] \\
& =\mathfrak{W}_{1}\left(\mathfrak{A}\left(g_{p} ; a\right) ; \partial \Lambda, \partial \Omega\right),
\end{aligned}
$$

for any $p=1,2, \ldots$ If $T_{\alpha}(a)$ is replaced with $S_{\alpha}(a)$, then the same formula holds with the symbol a replaced by $\operatorname{Re} a$ on the right-hand side.

Note that for bounded domains $\Lambda$ formula (2.10) is just another way to write the asymptotics (2.9), see Proof of Theorem 2.5. On the other hand, for unbounded $\Lambda$ formula (2.10) is an independent result.

Theorems 2.2 and 2.3 are derived from Theorem 2.4 in the same way as in [6] for smooth domains, and we do not provide details. However the methods of [6] do not allow one to derive from Theorem 2.5 analogues of Theorems 2.2 or 2.3 for unbounded domains $\Lambda$. This generalization will be done in another publication.

The main focus of the rest of this paper is on the proof of Theorems 2.4 and 2.5 .

\section{Auxiliary Results}

Here we collect some trace estimates and asymptotic formulas from [6] and [7] used in the proofs. The trace estimates established in [6] required that $\Lambda$ and $\Omega$ be $C^{1}$-smooth domains. In [7] most of those estimates are proved under the Lipschitz assumption only. On the other hand, the article [7] does not duplicate [6], and thus in the current article some of the estimates from [6] are re-proved for Lipschitz domains.

\subsection{Notation: Smooth Symbols}

In order to allow consideration of symbols $b=b(\mathbf{x}, \boldsymbol{\xi})$ with different scaling properties, we define for any $\ell, \rho>0$ the norms

$$
\mathbf{N}^{(n, m)}(b ; \ell, \rho)=\max _{\substack{0 \leq k \leq n \\ 0 \leq r \leq m}} \operatorname{ess~sup}_{\mathbf{x}, \boldsymbol{\xi}} \ell^{k} \rho^{r}\left|\nabla_{\mathbf{x}}^{k} \nabla_{\boldsymbol{\xi}}^{r} b(\mathbf{x}, \boldsymbol{\xi})\right|,
$$

with $n, m=0,1, \ldots$. If the norm (3.1) is finite for some (and hence for all) $\ell, \rho>0$ then we say that the symbol $b$ belongs to the class $\mathbf{S}^{(n, m)}$.

Below we often assume that various symbols $b=b(\mathbf{x}, \boldsymbol{\xi})$ are compactly supported, and the choice of the parameters $\ell, \rho$ in (3.1) is coordinated with the size of support. Precisely, we suppose that

$$
b \text { is supported on } B(\mathbf{z}, \ell) \times B(\boldsymbol{\mu}, \rho),
$$

with some $\mathbf{z}, \boldsymbol{\mu} \in \mathbb{R}^{d}$.

In what follows most of the bounds are obtained under the assumption that $\alpha \ell \rho \geq \ell_{0}$ with some fixed positive number $\ell_{0}$. The constants featuring 
in all the estimates below are independent of the symbols involved as well as of the parameters $\mathbf{z}, \boldsymbol{\mu}, \alpha, \ell, \rho$ but may depend on the constant $\ell_{0}$.

We begin with some natural estimates for smooth symbols. The notation $\mathfrak{S}_{1}$ is used for the trace class, and $\|\cdot\|_{\mathfrak{S}_{1}}$-for the trace class norm.

Proposition 3.1. Let $a, b \in \mathbf{S}^{(d+1, d+2)}$ be some symbols, and suppose that $b$ satisfies (3.2). Assume that $\alpha \ell \rho \geq \ell_{0}$. Then for $k=[d / 2]+1$ :

$$
\begin{aligned}
\left\|\mathrm{Op}_{\alpha}^{1}(a)\right\|+\left\|\mathrm{Op}_{\alpha}^{\mathrm{r}}(a)\right\| \lambda e C \mathrm{~N}^{(k, d+1)}(a, \ell, \rho), \\
\left\|\mathrm{Op}_{\alpha}^{1}(a)-\mathrm{Op}_{\alpha}^{\mathrm{r}}(a)\right\| \leq C(\alpha \ell \rho)^{-1} \mathrm{~N}^{(k, d+2)}(a, \ell, \rho),
\end{aligned}
$$

and

$$
\begin{aligned}
\left\|\mathrm{Op}_{\alpha}^{1}(b)\right\|_{\mathfrak{S}_{1}} \leq & C(\alpha \ell \rho)^{d} \mathbf{N}^{(d+1, d+1)}(b ; \ell, \rho), \\
\left\|\mathrm{Op}_{\alpha}^{1}(b)-\mathrm{Op}_{\alpha}^{\mathrm{r}}(b)\right\|_{\mathfrak{S}_{1}} \leq & C(\alpha \ell \rho)^{d-1} \mathrm{~N}^{(d+1, d+2)}(b ; \ell, \rho), \\
\left\|\mathrm{Op}_{\alpha}^{1}(a) \mathrm{Op}_{\alpha}^{1}(b)-\mathrm{Op}_{\alpha}^{1}(a b)\right\|_{\mathfrak{S}_{1}} \leq & C(\alpha \ell \rho)^{d-1} \mathrm{~N}^{(d+1, d+2)}(a ; \ell, \rho) \\
& \mathbf{N}^{(d+1, d+2)}(b ; \ell, \rho) .
\end{aligned}
$$

The boundedness of the operators $\mathrm{Op}_{\alpha}^{1}, \mathrm{Op}_{\alpha}^{\mathrm{r}}$ is a classical fact, and it can be found, e.g. in [1], Theorem $B_{1}^{\prime}$, where it was established under somewhat weaker smoothness assumptions. For the other estimates see [6], Lemmas 3.10-3.12 and Corollary 3.13.

\subsection{Bounds for Basic Domains}

Theorems 2.4 and 2.5 will be deduced from the asymptotics of "local" traces of the form $\operatorname{tr}\left(\mathrm{Op}_{\alpha}^{1}(b) g_{p}\left(T_{\alpha}(a)\right)\right), g_{p}(t)=t^{p}, p=1,2, \ldots$, with a compactly supported symbol $b$. In this section we concentrate on such "localized" operators. In fact, due to the bound (3.3) it will be unimportant which of the operators $\mathrm{Op}_{\alpha}^{\mathrm{l}}(b)$ or $\mathrm{Op}_{\alpha}^{\mathrm{r}}(b)$ is used for this localization. Thus we often use the notation $\mathrm{Op}_{\alpha}(b)$ to denote any of these two operators.

First we obtain some bounds for the case when both domains $\Lambda$ and $\Omega$ are basic Lipschitz, i.e. $\Lambda=\Gamma(\Phi)$ and $\Omega=\Gamma(\Psi)$ with some uniformly Lipschitz functions $\Phi$ and $\Psi$. The choice of Cartesian coordinates for which $\Lambda$ or $\Omega$ have the form (2.1) is not assumed to be the same for both domains. The constants in the estimates below depend only on the Lipschitz constants $M_{\Phi}, M_{\Psi}$ for the functions $\Phi$ and $\Psi$, and not on any other properties of the domains.

First one needs the following commutator estimates.

Proposition 3.2. Let $\Lambda, \Omega$ be basic Lipschitz domains. Let the symbol $b \in$ $\mathbf{S}^{(d+2, d+2)}$ satisfy (3.2). Assume that $\alpha \ell \rho \geq \ell_{0}$. Then

$$
\left\|\left[\mathrm{Op}_{\alpha}(b), P_{\Omega, \alpha}\right]\right\|_{\mathfrak{S}_{1}}+\left\|\left[\mathrm{Op}_{\alpha}(b), \chi_{\Lambda}\right]\right\|_{\mathfrak{S}_{1}} \leq C(\alpha \ell \rho)^{d-1} \mathrm{~N}^{(d+2, d+2)}(b ; \ell, \rho) .
$$

See [7], Remark 4.3.

Using these commutator estimates we can now reduce the problem to the operator $T_{\alpha}(1)$. 
Lemma 3.3. Let each of the domains $\Lambda$ and $\Omega$ be either a basic Lipschitz domain, or $\mathbb{R}^{d}$. Let $a, b \in \mathbf{S}^{(d+2, d+2)}$, and assume that $b$ satisfies (3.2). Let $\alpha \ell \rho \geq \ell_{0}$. Then

$$
\begin{aligned}
& \left\|\mathrm{Op}_{\alpha}(b) g_{p}\left(T_{\alpha}(a)\right)-\mathrm{Op}_{\alpha}\left(a^{p} b\right) g_{p}\left(T_{\alpha}(1)\right)\right\|_{\mathfrak{S}_{1}} \\
& \quad \leq C_{p}(\alpha \ell \rho)^{d-1} \mathrm{~N}^{(d+2, d+2)}(b ; \ell, \rho)\left(\mathrm{N}^{(d+2, d+2)}(a ; \ell, \rho)\right)^{p},
\end{aligned}
$$

for any $p=1,2, \ldots$ The same bound holds if one replaces $T_{\alpha}$ with $S_{\alpha}$.

Proof. Without loss of generality assume that $\mathbf{z}=\boldsymbol{\mu}=\mathbf{0}$ and that the $\mathrm{N}$ norms on the right-hand side of (3.4) equal 1. Let $\zeta, \eta \in \mathrm{C}_{0}^{\infty}\left(\mathbb{R}^{d}\right)$ be functions $\zeta=\zeta(\mathbf{x}), \eta=\eta(\boldsymbol{\xi})$ supported in the balls $B(\mathbf{0}, 2 \ell)$ and $B(\mathbf{0}, 2 \rho)$ respectively such that $b \zeta \eta=b$, and such that

$$
\ell^{n}\left|\nabla_{\mathbf{x}}^{n} \zeta(\mathbf{x})\right|+\rho^{n}\left|\nabla_{\boldsymbol{\xi}}^{n} \eta(\boldsymbol{\xi})\right| \leq \tilde{C}_{n}, \quad n=0,1, \ldots
$$

Represent $b=b(\zeta \eta)^{p}$ and commute the symbol $(\zeta(\mathbf{x}) \eta(\boldsymbol{\xi}))^{p}$ to the right using repeatedly Propositions 3.1 and 3.2:

$$
\left\|\mathrm{Op}_{\alpha}(b) g_{p}\left(T_{\alpha}(a)\right)-\mathrm{Op}_{\alpha}(b) g_{p}\left(T_{\alpha}(\zeta \eta a)\right)\right\|_{\mathfrak{S}_{1}} \leq C_{p}(\alpha \ell \rho)^{d-1} .
$$

The same bound holds if $T_{\alpha}$ is replaced with $S_{\alpha}$. Now, commuting $\zeta \eta a$ to the left, with the help of Propositions 3.1 and 3.2 again we arrive at (3.4).

Proposition 3.4. Let $\Lambda, \Omega$ be basic Lipschitz domains. Suppose that the symbol $b \in \mathbf{S}^{(d+2, d+2)}$ satisfies (3.2), and that $\alpha \ell \rho \geq 2$. Then

$$
\left\|\chi_{\Lambda} \mathrm{Op}_{\alpha}(b) P_{\Omega, \alpha}\left(1-\chi_{\Lambda}\right)\right\|_{\mathfrak{S}_{1}} \leq C(\alpha \ell \rho)^{d-1} \log (\alpha \ell \rho) \mathrm{N}^{(d+2, d+2)}(b ; \ell, \rho) .
$$

See [7], Theorem 4.6 .

Here is a useful consequence of the above bound:

Corollary 3.5. Under the conditions of Proposition 3.4,

$$
\left\|\mathrm{Op}_{\alpha}(b) T_{\alpha}(1)\left(I-T_{\alpha}(1)\right)\right\|_{\mathfrak{S}_{1}} \leq C(\alpha \ell \rho)^{d-1} \log (\alpha \ell \rho) \mathrm{N}^{(d+2, d+2)}(b ; \ell, \rho) .
$$

Proof. Without loss of generality assume that $\mathrm{N}^{(d+2, d+2)}(b ; \ell, \rho)=1$. Calculate:

$$
T_{\alpha}(1)\left(I-T_{\alpha}(1)\right)=\chi_{\Lambda} P_{\Omega, \alpha}\left(1-\chi_{\Lambda}\right) P_{\Omega, \alpha} \chi_{\Lambda},
$$

so that

$$
\begin{aligned}
& \left\|\mathrm{Op}_{\alpha}(b) T_{\alpha}(1)\left(I-T_{\alpha}(1)\right)\right\|_{\mathfrak{S}_{1}} \\
& \quad \leq\left\|\left[\mathrm{Op}_{\alpha}(b), \chi_{\Lambda}\right]\right\|_{\mathfrak{S}_{1}}+\left\|\chi_{\Lambda} \mathrm{Op}_{\alpha}(b) P_{\Omega, \alpha}\left(1-\chi_{\Lambda}\right)\right\|_{\mathfrak{S}_{1}}
\end{aligned}
$$

Propositions 3.2 and 3.4 lead to (3.6). 


\subsection{Bounds and Asymptotics for More General Domains}

The next group of results expresses the fact that the local asymptotics are determined by local properties of the boundaries $\partial \Lambda, \partial \Omega$. This is the key idea in the proof of Theorem 2.4. Let $\Lambda, \Omega$ and $\Lambda_{0}, \Omega_{0}$ be two pairs of domains such that each of $\Lambda_{0}, \Omega_{0}$ is either

1. a basic Lipschitz domain, or

2. the entire space $\mathbb{R}^{d}$, or

3. the empty set.

Suppose that

$$
\Lambda \cap B(\mathbf{z}, \ell)=\Lambda_{0} \cap B(\mathbf{z}, \ell), \quad \Omega \cap B(\boldsymbol{\mu}, \rho)=\Omega_{0} \cap B(\boldsymbol{\mu}, \rho) .
$$

The next localization result is crucial.

Lemma 3.6. Let $a, b \in \mathbf{S}^{(d+2, d+2)}$, and let $b$ satisfy (3.2). Suppose that the domains $\Lambda, \Omega$ and $\Lambda_{0}, \Omega_{0}$ are as specified above, and that $\alpha \ell \rho \geq \ell_{0}$. Then

$\left\|\left[\mathrm{Op}_{\alpha}(b), P_{\Omega, \alpha}\right]\right\|_{\mathfrak{S}_{1}}+\left\|\left[\mathrm{Op}_{\alpha}(b), \chi_{\Lambda}\right]\right\|_{\mathfrak{S}_{1}} \leq C(\alpha \ell \rho)^{d-1} \mathrm{~N}^{(d+2, d+2)}(b ; \ell, \rho)$,

and

$$
\begin{aligned}
& \left\|\mathrm{Op}_{\alpha}(b)\left(g_{p}\left(T_{\alpha}(a ; \Lambda, \Omega)\right)-g_{p}\left(T_{\alpha}\left(a ; \Lambda_{0}, \Omega_{0}\right)\right)\right)\right\|_{\mathfrak{S}_{1}} \\
& \quad \leq C(\alpha \ell \rho)^{d-1} \mathbf{N}^{(d+2, d+2)}(b ; \ell, \rho)\left(\mathrm{N}^{(d+2, d+2)}(a ; \ell, \rho)\right)^{p} .
\end{aligned}
$$

The same bound holds if $T_{\alpha}$ is replaced with $S_{\alpha}$.

For $C^{1}$-domains $\Lambda, \Omega$ estimates of this type were established in [6], Section 7. Generalization to the Lipschitz domains is quite straightforward and we present a proof here for the sake of completeness.

Proof of Lemma 3.6. Without loss of generality assume that the both Nnorms on the right-hand sides of (3.8) and (3.9) equal 1. For any two operators $A_{1}$ and $A_{2}$ we write $A_{1} \sim A_{2}$ if $\left\|A_{1}-A_{2}\right\|_{\mathfrak{S}_{1}} \leq C(\alpha \ell \rho)^{d-1}$, with a constant $C$ independent of $\alpha, \ell, \rho$.

Assume that $\Lambda_{0}, \Omega_{0}$ are basic Lipschitz domains. The following relations are consequences of (3.3) and Proposition 3.2:

$$
\mathrm{Op}_{\alpha}(b) \chi_{\Lambda} \sim \mathrm{Op}_{\alpha}^{\mathrm{r}}(b) \chi_{\Lambda_{0}} \sim \chi_{\Lambda_{0}} \mathrm{Op}_{\alpha}^{\mathrm{r}}(b) \sim \chi_{\Lambda_{0}} \mathrm{Op}_{\alpha}(b) .
$$

Taking the adjoints we also get $\chi_{\Lambda} \mathrm{Op}_{\alpha}(b) \sim \mathrm{Op}_{\alpha}(b) \chi_{\Lambda_{0}}$. In the same way one obtains similar relations for $P_{\Omega, \alpha}$ :

$$
\mathrm{Op}_{\alpha}(b) P_{\Omega, \alpha} \sim P_{\Omega_{0}, \alpha} \mathrm{Op}_{\alpha}(b), \quad P_{\Omega, \alpha} \mathrm{Op}_{\alpha}(b) \sim \mathrm{Op}_{\alpha}(b) P_{\Omega_{0}, \alpha} .
$$

Thus by Proposition 3.2,

$$
\left\{\begin{array}{l}
{\left[\mathrm{Op}_{\alpha}(b), \chi_{\Lambda}\right] \sim\left[\mathrm{Op}_{\alpha}(b), \chi_{\Lambda_{0}}\right] \sim 0,} \\
{\left[\mathrm{Op}_{\alpha}(b), P_{\Omega, \alpha}\right] \sim\left[\mathrm{Op}_{\alpha}(b), P_{\Omega_{0}, \alpha}\right] \sim 0 .}
\end{array}\right.
$$

If $\Lambda_{0}$ or $\Omega_{0}$ are either $\mathbb{R}^{d}$ or $\varnothing$, then the above relations hold for trivial reasons. This proves (3.8). 
Applying repeatedly the relations (3.10) and (3.11) in combination with Proposition 3.1 we arrive at

$$
\mathrm{Op}_{\alpha}(b)\left(T_{\alpha}(a ; \Lambda, \Omega)\right)^{p} \sim\left(T_{\alpha}\left(a ; \Lambda_{0}, \Omega_{0}\right)\right)^{p} \mathrm{Op}_{\alpha}(b) \sim \mathrm{Op}_{\alpha}(b)\left(T_{\alpha}\left(a ; \Lambda_{0}, \Omega_{0}\right)\right)^{p} .
$$

This relation coincides with (3.9).

The same argument leads to the bound of the form (3.9) for the operator $S_{\alpha}$

Lemma 3.7. Let $a, b \in \mathbf{S}^{(d+2, d+2)}$, and assume that $b$ satisfies (3.2). Let $\alpha \ell \rho \geq$ $\ell_{0}$. Suppose that $\Lambda$ and $\Omega$ satisfy (3.7), and one of the following two conditions is satisfied:

1. $\Lambda_{0}=\varnothing$ or $\Lambda_{0}=\mathbb{R}^{d}$,

2. $\Omega_{0}=\varnothing$ or $\Omega_{0}=\mathbb{R}^{d}$.

Then

$$
\begin{aligned}
& \left|\operatorname{tr}\left(\mathrm{Op}_{\alpha}(b) g_{p}\left(T_{\alpha}(a)\right)\right)-\alpha^{d} \mathfrak{W}_{0}\left(b g_{p}(a)\right)\right| \\
& \quad \leq C_{p}(\alpha \ell \rho)^{d-1} \mathbf{N}^{(d+2, d+2)}(b ; \ell, \rho)\left(\mathbf{N}^{(d+2, d+2)}(a ; \ell, \rho)\right)^{p} .
\end{aligned}
$$

Proof. By Lemmas 3.6 and 3.3 we may assume that $\Lambda=\Lambda_{0}, \Omega=\Omega_{0}$ and $a \equiv$ 1. Under any of the conditions of the lemma we have either $T_{\alpha}\left(1 ; \Lambda_{0}, \Omega_{0}\right)=0$ or $\chi_{\Lambda_{0}}$ or $P_{\Omega_{0}, \alpha}$. In the first case the left-hand side of (3.12) equals zero, and there is nothing to prove. If $T_{\alpha}(1)=\chi_{\Lambda_{0}}$, then the sought trace has the form $\operatorname{tr}\left(\mathrm{Op}_{\alpha}(b) \chi_{\Lambda_{0}}\right)$. This trace is easily found by integrating the kernel of the operator over the diagonal, and it does not depend on the choice of quantization. This immediately leads to (3.12). If $T_{\alpha}(1)=P_{\Omega_{0}, \alpha}$, then computing the trace $\operatorname{tr}\left(\mathrm{Op}_{\alpha}^{1}\left(b \chi_{\Omega_{0}}\right)\right)$ we obtain (3.12) again. Note that in this case it is convenient to choose the l-quantization for $\mathrm{Op}_{\alpha}(b)$.

The next result is also useful.

Lemma 3.8. Let the symbols $a, b$ be as in Lemma 3.7, and let $\alpha \ell \rho \geq \ell_{0}$. Suppose that $\Lambda$ and $\Omega$ satisfy (3.7). Then

$$
\begin{aligned}
& \left|\operatorname{tr}\left(\mathrm{Op}_{\alpha}(b) \chi_{\Lambda} g_{p}\left(T_{\alpha}\left(a ; \mathbb{R}^{d}, \Omega\right)\right) \chi_{\Lambda}\right)-\alpha^{d} \mathfrak{W}_{0}\left(b g_{p}(a) ; \Lambda, \Omega\right)\right| \\
& \quad \leq C_{p}(\alpha \ell \rho)^{d-1} \mathrm{~N}^{(d+2, d+2)}(b ; \ell, \rho)\left(\mathrm{N}^{(d+2, d+2)}(a ; \ell, \rho)\right)^{p} .
\end{aligned}
$$

Proof. Due to (3.8), the problem reduces to finding the trace of the operator

$$
\chi_{\Lambda} \mathrm{Op}_{\alpha}^{\mathrm{l}}(b) g_{p}\left(T_{\alpha}\left(a ; \mathbb{R}^{d}, \Omega\right)\right) \chi_{\Lambda} .
$$

As in the proof of Lemma 3.7, by virtue of Lemmas 3.6 and 3.3 we may assume that $\Omega=\Omega_{0}$ and $a \equiv 1$. Thus $T_{\alpha}\left(1 ; \mathbb{R}^{d}, \Omega_{0}\right)=P_{\Omega_{0}, \alpha}$. Again, the trace of the operator $\chi_{\Lambda} \mathrm{Op}_{\alpha}^{\mathrm{l}}(b) P_{\Omega_{0}, \alpha} \chi_{\Lambda}$ is easily seen to be equal to $\alpha^{d} \mathfrak{W}_{0}(b ; \Lambda, \Omega)$.

So far it was enough to assume that the domains were Lipschitz. To state the asymptotic result we need more restrictive conditions. 
Proposition 3.9. Let $a, b \in \mathbf{S}^{(d+2, d+2)}$, and let $b$ satisfy (3.2). Assume that (3.7) holds with some basic domains $\Lambda_{0}, \Omega_{0}$ such that $\Lambda_{0}$ is $C^{1}$ and $\Omega_{0}$ is $C^{3}$. Then

$$
\begin{aligned}
\operatorname{tr}\left(\mathrm{Op}_{\alpha}^{1}(b) g_{p}\left(T_{\alpha}(a)\right)\right)= & \alpha^{d} \mathfrak{W}_{0}\left(b g_{p}(a) ; \Lambda, \Omega\right) \\
& +\alpha^{d-1} \log \alpha \mathfrak{W}_{1}\left(b \mathfrak{A}\left(g_{p} ; a\right) ; \partial \Lambda, \partial \Omega\right)+o\left(\alpha^{d-1} \log \alpha\right),
\end{aligned}
$$

as $\alpha \rightarrow \infty$.

This proposition follows from [6], Theorem 11.1 upon application of Lemma 3.6.

\section{Proof of the Main Theorems}

Here we concentrate on proving Theorems 2.4 and 2.5. As explained earlier, Theorem 2.4 implies the main results - Theorems 2.2 and 2.3.

\subsection{An Intermediate Local Asymptotics}

We begin with the following local result:

Theorem 4.1. Suppose that $b \in \mathbf{S}^{(d+2, d+2)}$ is a symbol with compact support in both variables, and that $\Lambda$ is a piece-wise $\mathrm{C}^{1}$ basic domain, and $\Omega$ a piece-wise $\mathrm{C}^{3}$ basic domain. Then

$$
\begin{aligned}
\operatorname{tr}\left(\mathrm{Op}_{\alpha}^{1}(b) g_{p}\left(T_{\alpha}(1)\right)\right)= & \alpha^{d} \mathfrak{W}_{0}(b ; \Lambda, \Omega)+\alpha^{d-1} \log \alpha \mathfrak{W}_{1}\left(b \mathfrak{A}\left(g_{p} ; 1\right) ; \partial \Lambda, \partial \Omega\right) \\
& +o\left(\alpha^{d-1} \log \alpha\right),
\end{aligned}
$$

as $\alpha \rightarrow \infty$.

Without loss of generality assume that the symbol $b$ is supported on $B(\mathbf{0}, 1) \times B(\mathbf{0}, 1)$. If $B(\mathbf{0}, 1) \cap \partial \Lambda=\varnothing$ or $B(\mathbf{0}, 1) \cap \partial \Omega=\varnothing$, then the required asymptotics immediately follow from Lemma 3.7. Assume that neither of the above intersections is empty. Cover the boundaries $\partial \Lambda \cap B(\mathbf{0}, 1)$ and $\partial \Omega \cap B(\mathbf{0}, 1)$ with finitely many open balls of radius $\varepsilon>0$. Denote the number of such balls by $J=J_{\varepsilon}$ and $K=K_{\varepsilon}$ respectively. Since $\partial \Lambda$ and $\partial \Omega$ are Lipschitz, one can construct these coverings in such a way that the number of intersections of each ball with the other ones is bounded from above uniformly in $\varepsilon$ and

$$
J_{\varepsilon}, K_{\varepsilon} \leq C \varepsilon^{1-d} .
$$

Let $\Sigma_{\Lambda}\left(\right.$ resp. $\left.\Sigma_{\Omega}\right)$ be the set of indices $j$ (resp. $k$ ) such that the ball from the constructed covering indexed $j$ (resp. $k$ ) has a non-empty intersection with the set $(\partial \Lambda)_{\mathrm{s}}\left(\operatorname{resp} .(\partial \Omega)_{\mathrm{s}}\right)$. Since the sets $(\partial \Lambda)_{\mathrm{s}},(\partial \Omega)_{\mathrm{s}}$ are built out of Lipschitz surfaces, by construction of the covering we have

$$
\#\left(\Sigma_{\Lambda}\right), \#\left(\Sigma_{\Omega}\right) \leq C \varepsilon^{2-d} .
$$

We may assume that the covering balls with indices $j \notin \Sigma_{\Lambda}$ (resp. $\left.k \notin \Sigma_{\Omega}\right)$ are separated from $(\partial \Lambda)_{\mathrm{s}}\left(\right.$ resp. $\left.(\partial \Omega)_{s}\right)$. Thus in each of these balls the boundary $\partial \Lambda($ resp. $\partial \Omega)$ is $C^{1}\left(\right.$ resp. $\left.C^{3}\right)$. 
Denote by $\phi_{j}, j=1,2, \ldots, J$, and $\psi_{k}, k=1,2, \ldots, K$ the associated smooth partitions of unity, so that the functions

$$
\phi(\mathbf{x}):=\sum_{j=1}^{J} \phi_{j}(\mathbf{x}), \quad \psi(\boldsymbol{\xi}):=\sum_{j=1}^{K} \psi_{k}(\boldsymbol{\xi})
$$

equal 1 on a neighbourhood of $\partial \Lambda \cap B(\mathbf{0}, 1)$ and $\partial \Omega \cap B(\mathbf{0}, 1)$ respectively, and

$$
\left|\nabla^{n} \phi_{j}(\mathbf{x})\right|+\left|\nabla^{n} \psi_{j}(\boldsymbol{\xi})\right| \leq C_{n} \varepsilon^{-n}, \quad n=0,1, \ldots,
$$

uniformly in $\mathbf{x}$ and $\boldsymbol{\xi}$. The symbol $b(1-\phi \psi)$ is supported away from $(\partial \Lambda \cap$ $B(\mathbf{0}, 1)) \times(\partial \Omega \cap B(\mathbf{0}, 1))$. Thus Lemma 3.7 implies that

$$
\left|\operatorname{tr}\left(\operatorname{Op}_{\alpha}^{1}(b(1-\phi \psi)) g_{p}\left(T_{\alpha}(1)\right)\right)-\alpha^{d} \mathfrak{W}_{0}(b(1-\phi \psi))\right| \leq C_{\varepsilon} \alpha^{d-1} .
$$

The constant $C_{\varepsilon}$ on the right-hand side depends on the symbol $b$, and on $\varepsilon$, but the latter fact does not matter for the rest of the proof. It remains to study the trace $\operatorname{tr}\left(\mathrm{Op}_{\alpha}^{\mathrm{l}}(b \phi \psi) g_{p}\left(T_{\alpha}(1)\right)\right)$.

Let us separate contributions from the smooth and singular parts of the boundaries $\partial \Lambda$ and $\partial \Omega$. Denote

$$
\tilde{b}(\mathbf{x}, \boldsymbol{\xi})=\sum_{j \notin \Sigma_{\Lambda}} \sum_{k \notin \Sigma_{\Omega}} b_{j k}(\mathbf{x}, \boldsymbol{\xi}), b_{j k}(\mathbf{x}, \boldsymbol{\xi})=b(\mathbf{x}, \boldsymbol{\xi}) \phi_{j}(\mathbf{x}) \psi_{k}(\boldsymbol{\xi}) .
$$

The support of $\tilde{b}$ contains only smooth parts of the boundaries $\partial \Lambda$ and $\partial \Omega$, so by Proposition 3.9 we have

$$
\begin{aligned}
& \lim _{\alpha \rightarrow \infty} \frac{1}{\alpha^{d-1} \log \alpha}\left(\operatorname{tr}\left(\operatorname{Op}_{\alpha}^{1}(\tilde{b}) g_{p}\left(T_{\alpha}(1)\right)\right)-\alpha^{d} \mathfrak{W}_{0}(\tilde{b})\right. \\
& \left.-\alpha^{d-1} \log \alpha \mathfrak{W}_{1}\left(\tilde{b} \mathfrak{A}\left(g_{p} ; 1\right)\right)\right)=0
\end{aligned}
$$

It remains to handle the cases when $j \in \Sigma_{\Lambda}$ or $k \in \Sigma_{\Omega}$. Let

$$
\boldsymbol{\Sigma}=\left\{(j, k): j \in \Sigma_{\Lambda} \text { or } k \in \Sigma_{\Omega}\right\} .
$$

Lemma 4.2. Let $b_{j k}$ be as defined above, and let $p \geq 1$. Then

$$
\begin{aligned}
& \lim \sup \frac{1}{\alpha^{d-1} \log \alpha} \sum_{(j, k) \in \boldsymbol{\Sigma}} \mid \operatorname{tr}\left(\operatorname{Op}_{\alpha}^{1}\left(b_{j k}\right) g_{p}\left(T_{\alpha}(1)\right)\right) \\
& -\alpha^{d} \mathfrak{W}_{0}\left(b_{j k}\right)-\alpha^{d-1} \log \alpha \mathfrak{W}_{1}\left(b_{j k} \mathfrak{A}\left(g_{p} ; 1\right)\right) \mid \leq C \varepsilon,
\end{aligned}
$$

as $\alpha \rightarrow \infty$.

Proof. It is enough to establish the estimate

$$
\begin{aligned}
& \limsup _{\alpha \rightarrow \infty} \frac{1}{\alpha^{d-1} \log \alpha} \mid \operatorname{tr}\left(\operatorname{Op}_{\alpha}^{1}\left(b_{j k}\right) g_{p}\left(T_{\alpha}(1)\right)\right) \\
& \quad-\alpha^{d} \mathfrak{W}_{0}\left(b_{j k}\right)-\alpha^{d-1} \log \alpha \mathfrak{W}_{1}\left(b_{j k} \mathfrak{A}\left(g_{p} ; 1\right)\right) \mid \leq C \varepsilon^{2(d-1)} .
\end{aligned}
$$


Indeed, in view of (4.2) and (4.3), the number of summands on the lefthand side of (4.7) does not exceed $C \varepsilon^{3-2 d}$, and hence summing (4.8) up over $(j, k) \in \boldsymbol{\Sigma}$ we obtain (4.7). If $p=1$, then the trace asymptotics of the operator $\mathrm{Op}_{\alpha}^{1}\left(b_{j k}\right) T_{\alpha}(1)$ are easy to find. Indeed, by Lemma 3.8 , we have

$$
\operatorname{tr}\left(\mathrm{Op}_{\alpha}^{1}\left(b_{j k}\right) \chi_{\Lambda} P_{\Omega, \alpha} \chi_{\Lambda}\right)=\alpha^{d} \mathfrak{W}_{0}\left(b_{j k}\right)+O\left(\left(\alpha \varepsilon^{2}\right)^{d-1}\right) .
$$

Thus it remains to study the trace of the operator

$$
\mathrm{Op}_{\alpha}^{1}\left(b_{j k}\right) g\left(T_{\alpha}(1)\right), \quad g(t)=t^{p}-t,
$$

with $p \geq 2$. Represent $g(t)=t(1-t) \tilde{g}(t)$ with a polynomial $\tilde{g}$, and estimate using (3.6):

$$
\begin{aligned}
\left\|\mathrm{Op}_{\alpha}^{1}\left(b_{j k}\right) g\left(T_{\alpha}(1)\right)\right\|_{\mathfrak{S}_{1}} & \leq\left\|\operatorname{Op}_{\alpha}^{1}\left(b_{j k}\right) T_{\alpha}(1)\left(I-T_{\alpha}(1)\right)\right\|_{\mathfrak{S}_{1}}\left\|\tilde{g}\left(T_{\alpha}(1)\right)\right\| \\
& \leq C \max _{0 \leq t \leq 1}|\tilde{g}(t)|\left(\alpha \varepsilon^{2}\right)^{d-1} \log \left(\alpha \varepsilon^{2}\right),
\end{aligned}
$$

for sufficiently large $\alpha$. Together with (4.9) this implies that

$$
\limsup \frac{1}{\alpha^{d-1} \log \alpha}\left|\operatorname{tr}\left(\mathrm{Op}_{\alpha}^{1}\left(b_{j k}\right) g_{p}\left(T_{\alpha}(1)\right)\right)-\alpha^{d} \mathfrak{W}_{0}\left(b_{j k}\right)\right| \leq C \varepsilon^{2(d-1)},
$$

as $\alpha \rightarrow \infty$. It follows straight from the definition (2.4) that

$$
\left|\mathfrak{W}_{1}\left(b_{j k} \mathfrak{A}\left(g_{p} ; 1\right)\right)\right| \leq C \varepsilon^{2(d-1)},
$$

so (4.10) entails (4.8), as claimed.

Proof of Theorem 4.1. Remembering that $\mathfrak{W}_{1}\left(b(1-\phi \psi) \mathfrak{A}\left(g_{p} ; 1\right)\right)=0$, and putting together (4.6), (4.5) and (4.7) we obtain that

$$
\begin{aligned}
& \lim \sup \frac{1}{\alpha^{d-1} \log \alpha} \mid \operatorname{tr}\left(\operatorname{Op}_{\alpha}^{1}(b) g_{p}\left(T_{\alpha}(1)\right)\right) \\
& -\alpha^{d} \mathfrak{W}_{0}(b)-\alpha^{d-1} \log \alpha \mathfrak{W}_{1}\left(b \mathfrak{A}\left(g_{p} ; 1\right)\right) \mid \leq C \varepsilon,
\end{aligned}
$$

as $\alpha \rightarrow \infty$, for any $\varepsilon>0$. Taking $\varepsilon \rightarrow 0$ we arrive at the asymptotics (4.1).

\subsection{Proof of Theorems 2.4 and 2.5}

The proofs amount to putting together local asymptotic formulas and estimates obtained above. The argument is based on partition of unity, and is rather standard. We present it for the sake of completeness. Also, all the proofs are conducted for the operator $T_{\alpha}$ only-the argument for $S_{\alpha}$ is essentially the same.

The next two lemmas are the last building blocks in the proofs of Theorems 2.4 and 2.5 .

Lemma 4.3. Let the conditions of Theorem 2.5 be satisfied. Let $h \in \mathrm{C}_{0}^{\infty}\left(\mathbb{R}^{d}\right)$ be an arbitrary function. Then 


$$
\begin{aligned}
& \lim _{\alpha \rightarrow \infty} \frac{1}{\alpha^{d-1} \log \alpha}\left[\operatorname{tr}\left(h g_{p}\left(T_{\alpha}(a ; \Lambda, \Omega)\right)\right)-\alpha^{d} \mathfrak{W}_{0}\left(h g_{p}(a) ; \Lambda, \Omega\right)\right] \\
& \quad=\mathfrak{W}_{1}\left(h \mathfrak{A}\left(g_{p} ; a\right) ; \partial \Lambda, \partial \Omega\right)
\end{aligned}
$$

and

$$
\begin{aligned}
\lim _{\alpha \rightarrow \infty} \frac{1}{\alpha^{d-1} \log \alpha} \operatorname{tr} & {\left[\operatorname{tr}\left(h \chi_{\Lambda} g_{p}\left(T_{\alpha}\left(a ; \mathbb{R}^{d}, \Omega\right)\right) \chi_{\Lambda}\right)\right.} \\
& \left.-\alpha^{d} \mathfrak{W}_{0}\left(h g_{p}(a) ; \Lambda, \Omega\right)\right]=0 .
\end{aligned}
$$

If $T_{\alpha}(a)$ is replaced with $S_{\alpha}(a)$, then the same formulas hold with the symbol a replaced by $\operatorname{Re} a$ in $\mathfrak{W}_{0}$ and $\mathfrak{W}_{1}$.

Proof. Let $R>0$ be such that $\operatorname{supp} h \in B(\mathbf{0}, R)$, and let either $\Lambda$ or $\mathbb{R}^{d} \backslash \Lambda$ be contained in $B(\mathbf{0}, R)$. Since the domains $\Lambda \cap B(\mathbf{0}, R)$ and $\Omega$ are bounded, we can cover their closures by finitely many open balls such that in each of them each domain $\Lambda$ or $\Omega$ is represented by a basic domain or by $\mathbb{R}^{d}$. Denote by $\left\{\phi_{j}\right\}$ and $\left\{\psi_{k}\right\}$ the partitions of unity subordinate to these coverings. Represent

$$
h \chi_{\Lambda} P_{\Omega, \alpha}=\sum_{j, k} \chi_{\Lambda} \mathrm{Op}_{\alpha}^{1}\left(b_{j k}\right) P_{\Omega, \alpha}, b_{j k}(\mathbf{x}, \boldsymbol{\xi})=h(\mathbf{x}) \phi_{j}(\mathbf{x}) \psi_{k}(\boldsymbol{\xi}) .
$$

Consequently, in order to prove (4.11) it suffices to find the sought asymptotics for the operator

$$
\chi_{\Lambda} \mathrm{Op}_{\alpha}^{1}\left(b_{j k}\right) P_{\Omega, \alpha} \mathrm{Op}_{\alpha}^{1}(a) P_{\Omega, \alpha}\left(T_{\alpha}(a ; \Lambda, \Omega)\right)^{p-1},
$$

for each $j$ and $k$. By virtue of (3.8) this is equivalent to studying the operator

$$
\mathrm{Op}_{\alpha}^{1}\left(b_{j k}\right)\left(T_{\alpha}(a ; \Lambda, \Omega)\right)^{p} .
$$

Now, due to (3.9), we can replace each domain $\Lambda$ or $\Omega$ by the appropriate basic domain or by $\mathbb{R}^{d}$. Furthermore, Lemma 3.3 ensures that the symbol $a$ can be replaced by the constant symbol $a \equiv 1$. Now Theorem 4.1 implies that

$$
\begin{aligned}
& \operatorname{tr} \mathrm{Op}_{\alpha}^{1}\left(b_{j k}\right) g_{p}\left(T_{\alpha}(1 ; \Lambda, \Omega)\right)=\alpha^{d} \mathfrak{W}_{0}\left(b_{j k} ; \Lambda, \Omega\right) \\
& \quad+\alpha^{d-1} \log \alpha \mathfrak{W}_{1}\left(b_{j k} \mathfrak{A}\left(g_{p} ; 1\right) ; \partial \Lambda, \partial \Omega\right)+o\left(\alpha^{d-1} \log \alpha\right),
\end{aligned}
$$

as $\alpha \rightarrow \infty$. Summing over $j$ and $k$ we obtain formula (4.11).

Similarly, for the asymptotics (4.12) it suffices to study the operator

$$
\mathrm{Op}_{\alpha}^{1}\left(b_{j k}\right) \chi_{\Lambda}\left(T_{\alpha}\left(a ; \mathbb{R}^{d}, \Omega\right)\right)^{p} \chi_{\Lambda} .
$$

By Lemma 3.8, the trace of this operator equals

$$
\alpha^{d} \mathfrak{W}_{0}\left(b_{j k} g_{p}(a) ; \Lambda, \Omega\right)+O\left(\alpha^{d-1}\right) .
$$

Summing over $j$ and $k$ we obtain formula (4.12), as required.

The following lemma concentrates on the case of unbounded $\Lambda$.

Lemma 4.4. Suppose that $a \in \mathbf{S}^{(d+2, d+2)}$. Let $\Omega$ and $\Lambda$ be Lipschitz domains such that $\Omega$ and $\mathbb{R}^{d} \backslash \Lambda$ are bounded. Let $h \in \mathrm{C}_{0}^{\infty}\left(\mathbb{R}^{d}\right)$ be a function such that $h(\mathbf{x})=1$ for all $\mathbf{x} \in \mathbb{R}^{d} \backslash \Lambda$. Then 


$$
\left\|(I-h)\left[g_{p}\left(T_{\alpha}(a, \Lambda, \Omega)\right)-g_{p}\left(T_{\alpha}\left(a, \mathbb{R}^{d}, \Omega\right)\right)\right]\right\|_{\mathfrak{S}_{1}} \leq C \alpha^{d-1},
$$

for any $p=1,2, \ldots$, with a constant $C$ independent of $\alpha$. The same bound holds if $T_{\alpha}$ is replaced with $S_{\alpha}$.

Proof. For brevity we write $T_{\alpha}(\Lambda)=T_{\alpha}(a ; \Lambda, \Omega)$. For any two operators $A_{1}$ and $A_{2}$ we write $A_{1} \sim A_{2}$ if $\left\|A_{1}-A_{2}\right\|_{\mathfrak{S}_{1}} \leq C \alpha^{d-1}$, with a constant $C$ independent of $\alpha$.

First we prove that

$$
(I-h)\left(T_{\alpha}(\Lambda)\right)^{p} \sim\left(T_{\alpha}\left(\mathbb{R}^{d}\right)\right)^{p}(I-h) .
$$

Suppose that $p=1$. Let $\eta \in \mathrm{C}_{0}^{\infty}\left(\mathbb{R}^{d}\right)$ be a function such that $\eta \chi_{\Omega}=\chi_{\Omega}$, and let $b(\mathbf{x}, \boldsymbol{\xi})=h(\mathbf{x}) \eta(\boldsymbol{\xi})$. Since $(1-h) \chi_{\Lambda}=1-h$, we have

$$
(I-h) T_{\alpha}(\Lambda)=T_{\alpha}\left(\mathbb{R}^{d}\right) \chi_{\Lambda}-\mathrm{Op}_{\alpha}^{1}(b) T_{\alpha}\left(\mathbb{R}^{d}\right) \chi_{\Lambda} .
$$

Using the partition of unity $\left\{\psi_{j}\right\}$ featuring in the proof of the previous lemma, and then bound (3.8) and Lemma 3.1, we can claim that

$$
\mathrm{Op}_{\alpha}^{1}(b) T_{\alpha}\left(\mathbb{R}^{d}\right) \chi_{\Lambda} \sim T_{\alpha}\left(\mathbb{R}^{d}\right) \mathrm{Op}_{\alpha}^{1}(b) \chi_{\Lambda} \sim T_{\alpha}\left(\mathbb{R}^{d}\right) \mathrm{Op}_{\alpha}^{\mathrm{r}}(b) \chi_{\Lambda}=T_{\alpha}\left(\mathbb{R}^{d}\right) h \chi_{\Lambda} .
$$

Together with (4.16) this gives (4.15) for $p=1$.

Suppose now that (4.15) holds for $p=k$, and let us prove it for $p=k+1$. Write:

$$
\begin{aligned}
(I- & h)\left(T_{\alpha}(\Lambda)\right)^{k+1}-\left(T_{\alpha}\left(\mathbb{R}^{d}\right)\right)^{k+1}(I-h) \\
= & {\left[(I-h)\left(T_{\alpha}(\Lambda)\right)^{k}-\left(T_{\alpha}\left(\mathbb{R}^{d}\right)\right)^{k}(I-h)\right] T_{\alpha}(\Lambda) } \\
& +\left(T_{\alpha}\left(\mathbb{R}^{d}\right)\right)^{k}\left[(I-h) T_{\alpha}(\Lambda)-T_{\alpha}\left(\mathbb{R}^{d}\right)(I-h)\right] .
\end{aligned}
$$

The sought bound follows from (4.15) for $p=1$ and $p=k$.

To conclude the proof write

$$
\begin{aligned}
(I-h)\left[\left(T_{\alpha}(\Lambda)\right)^{p}-\left(T_{\alpha}\left(\mathbb{R}^{d}\right)\right)^{p}\right]= & (I-h)\left(T_{\alpha}(\Lambda)\right)^{p}-\left(T_{\alpha}\left(\mathbb{R}^{d}\right)\right)^{p}(I-h) \\
& +\left[\left(T_{\alpha}\left(\mathbb{R}^{d}\right)\right)^{p}, I-h\right],
\end{aligned}
$$

so that (4.14) follows from (4.15) used twice: for the domain $\Lambda$ itself, and for $\Lambda=\mathbb{R}^{d}$.

Now we can proceed to the proof of Theorems 2.4 and 2.5. As explained earlier, the proofs are conducted only for the operators $T_{\alpha}$.

Proof of Theorem 2.4. Since $\Lambda$ is bounded, use formula (4.11) with a function $h \in \mathrm{C}_{0}^{\infty}\left(\mathbb{R}^{d}\right)$ such that $h \chi_{\Lambda}=\chi_{\Lambda}$. This completes the proof.

Proof of Theorem 2.5. If $\Lambda$ is bounded, then Theorem 2.5 follows from formulas (4.11) and (4.12) with a function $h$ as in the above proof of Theorem 2.4.

Suppose that $\mathbb{R}^{d} \backslash \Lambda$ is bounded. Let $h \in \mathrm{C}_{0}^{\infty}\left(\mathbb{R}^{d}\right)$ be a function such that $h(\mathbf{x})=1$ for $\mathbf{x} \in \mathbb{R}^{d} \backslash \Lambda$. Due to Lemma 4.4 it suffices to establish the formula

$$
\begin{aligned}
& \operatorname{tr}\left\{h\left[g_{p}\left(T_{\alpha}(\Lambda)\right)-\chi_{\Lambda} g_{p}\left(T_{\alpha}\left(\mathbb{R}^{d}\right)\right) \chi_{\Lambda}\right]\right\} \\
& \quad=\alpha^{d-1} \log \alpha \mathfrak{W}_{1}\left(\mathfrak{A}\left(g_{p} ; a\right) ; \Lambda, \Omega\right)+o\left(\alpha^{d-1} \log \alpha\right),
\end{aligned}
$$

where we have denoted $T_{\alpha}(\Lambda)=T_{\alpha}(a ; \Lambda, \Omega)$. But this formula immediately follows from (4.11) and (4.12) again. Thus the proof is complete. 
Theorems 2.2 and 2.3 are derived from Theorem 2.4 by approximating $g$ with polynomials, in the same way as in [6], Section 12.

\section{Acknowledgements}

The author is grateful to J. Oldfield and W. Spitzer for critical remarks. This work was supported by EPSRC Grant EP/J016829/1.

Open Access. This article is distributed under the terms of the Creative Commons Attribution License which permits any use, distribution, and reproduction in any medium, provided the original author(s) and the source are credited.

\section{References}

[1] Cordes, H.O.: On compactness of commutators of multiplications and convolutions, and boundedness of pseudodifferential operators. J. Funct. Anal. 18, 115$131(1975)$

[2] Gioev, D., Klich, I.: Entanglement entropy of fermions in any dimension and the Widom conjecture. Phys. Rev. Lett. 96(10), 100503 (2006)

[3] Helling, R.C., Leschke, H., Spitzer, W.L.: A special case of a conjecture by Widom with implications to fermionic entanglement entropy. Int. Math. Res. Not. 2011, 1451-1482 (2011)

[4] Leschke, H., Spitzer, W.L., Sobolev, A.V.: Scaling of Rényi entanglement entropies of the free Fermi-gas ground state: a rigorous proof. Phys. Rev. Lett. 112, 160403

[5] Robert, D.: Autour de l'Approximation Semi-Classique. Progress in Mathematics. Birkhäuser, Boston (1987)

[6] Sobolev, A.V.: Pseudo-differential operators with discontinuous symbols: Widom's conjecture. Mem. AMS 222(1043) (2013)

[7] Sobolev, A.V.: On the Schatten-von Neumann properties of some pseudodifferential operators. J. Funct. Anal. 266, 5886-5911 (2014)

[8] Widom, H.: On a Class of Integral Operators with Discontinuous Symbol, Toeplitz centennial (Tel Aviv, 1981), pp. 477-500. Operator Theory: Advances and Applications, vol. 4. Birkhäuser, Basel (1982)

[9] Widom, H.: Asymptotic Expansions for Pseudodifferential Operators on Bounded Domains. Lecture Notes in Mathematics, vol. 1152. Springer, Berlin (1985)

[10] Widom, H.: On a class of integral operators on a half-space with discontinuous symbol. J. Funct. Anal. 88(1), 166-193 (1990)
A. V. Sobolev
Department of Mathematics
University College London
Gower Street
London WC1E 6BT, UK
e-mail: a.sobolev@ucl.ac.uk

Received: August 11, 2014.

Revised: September 10, 2014. 